\title{
Simulation of electrically tunable semiconductor nanopores for ion current/single bio-molecule manipulation
}

\author{
Maria E. Gracheva · Jean-Pierre Leburton
}

Published online: 15 January 2008

(C) Springer Science+Business Media LLC 2008

\begin{abstract}
We show that a semiconductor membrane made of two thin layers of opposite ( $n$ - and $p$-) doping can perform electrically tunable ion current rectification and filtering in a nanopore. Our model is based on the solution of the 3D Poisson equation for the electrostatic potential in a double-cone nanopore, combined with a transport model. It predicts that for appropriate biasing of the membraneelectrolyte system, transitions from ohmic behavior to sharp rectification with vanishing leakage current are achievable. Further more, ion current rectifying and filtering regimes of the nanopore correspond to different charge states in the $p-n$ membrane which can be tuned with appropriate biasing of the $n$ - and $p$-layers.
\end{abstract}

Keywords Semiconductor membrane - Layered structure Diode $\cdot$ Ion filter $\cdot$ Single molecule manipulation

\section{Introduction}

Proteinaceous nanopores have been studied for the last decade for their essential role in biology as nanoscale channels regulating the ion flow through cell membranes as well

\section{M.E. Gracheva ( $\square)$}

Beckman Institute for Advanced Science and Technology,

University of Illinois (Urbana-Champaign),

405 N. Mathews Ave., Urbana, IL 61801, USA

e-mail: gracheva@uiuc.edu

\section{J.-P. Leburton}

Department of Electrical and Computer Engineering,

Beckman Institute for Advanced Science and Technology,

University of Illinois (Urbana-Champaign),

405 N. Mathews Ave., Urbana, IL 61801, USA

e-mail: jleburto@uiuc.edu as exhibiting ion selectivity [1,2]. Different types of biological channel exist, each of which is highly specialized, for example, to channel water or selectively allow entrance of ions and proteins into a cell. In the last few years, artificial nanopores in dielectric membranes etched by high energy ion or electron beams have been proposed as a substitute to biological ion channels [3-5]. Properties of the track-etched membranes have been studied in comparison to the properties of the various biological channels. Once created such artificial membranes usually have limited tunability since membrane charge selectivity is fixed. Also, such membranes are electrically insulating and do not provide tunable electrostatic control of the ion concentration inside or the ion flow through the nanopore [6, 7]. We propose to use membranes made of spatially separated $n$ - and $p$-type doping layers (Fig. 1) to electrically tune the potential landscape in the channel and modulate the ionic conductance through the nanopore.

\section{$2 p-n$ semiconductor membrane model}

The nanopore-membrane structure geometry is shown schematically in Fig. 1, and consists of two $12 \mathrm{~nm} \mathrm{Si} \mathrm{lay-}$ ers of different doping: the top layer is $n$-doped (typically $N_{d}^{n}=2 \times 10^{20} \mathrm{~cm}^{-3}$ ) and the bottom layer is $p$-doped typically $N_{d}^{p}=2 \times 10^{20} \mathrm{~cm}^{-3}$ ). The membrane is immersed in an electrolyte $\mathrm{KCl}$ solution, with a concentration varying from $0.01 \mathrm{M}$ to $1 \mathrm{M}$. We treat the ion charge distribution in the nanopore as a continuum for which the Poisson equation is solved self-consistently by a multigrid method [8] in the electrolyte-membrane system. We assume the ions in the electrolyte are fully dissociated and obey the Boltzmann distribution, whereas electrons and holes in the semiconductor are governed by the Fermi-Dirac statistics. We 


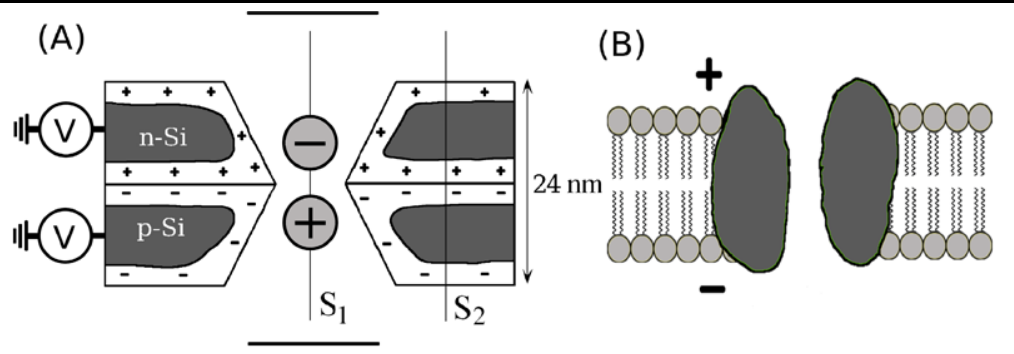

Fig. 1 (A) Geometry of the modeled nanopore in a solid-state membrane. The center $X-Y$ cross-section is shown (the center $Y-Z$ crosssection is similar). Two cuts $S_{1}$ and $S_{2}$ through the structure are in-

use virtual solid-state parameters for the solution which enables us to formulate an all-semiconductor model for the charge and electric potential in the electrolyte and solid-state materials [9]. The electrolyte/solid state and semiconductor/oxide interfaces are modeled by introducing a conduction band offset between materials at their interfaces, i.e.: $E_{c}^{\mathrm{SiO}_{2}}-E_{c}^{\mathrm{Si}}=3.2 \mathrm{eV}, E_{c}^{\text {solution }}-E_{c}^{\mathrm{Si}}=-0.3 \mathrm{eV}$. We model the bias potential applied to the membrane with respect to the electrolyte by varying the quasi-Fermi levels of the $\mathrm{Si}$ $n$ - $\left(V_{n}\right)$ and $p$ - $\left(V_{p}\right)$ sides of the membrane separately over the $-1 \mathrm{~V}$ to $1 \mathrm{~V}$ range. The whole surface of the nanoporemembrane is covered by a $8 \AA$ surface layer of $\mathrm{SiO}_{2}$ containing a fixed negative charge $\sigma$. The surface charge density was varied at first, but unless otherwise indicated the calculations are performed for $\sigma=-0.0256 \mathrm{C} / \mathrm{m}^{2}$. The membrane is immersed in an electrolyte $\mathrm{KCl}$ solution, with a concentration varying from $0.01 \mathrm{M}$ to $1 \mathrm{M}$. Each material is characterized by its relative permittivity, i.e., $\varepsilon_{\mathrm{Si}}=11.7$, $\varepsilon_{\mathrm{SiO}_{2}}=3.9$. The dielectric constant of the electrolyte solution is chosen $\varepsilon_{\text {electrolyte }}=78$. The model details are described in $[4,10]$.

\section{Ion current rectification and filtering}

In Fig. 2 anion and cation concentrations at the nanopore center are shown for a solution concentration $[\mathrm{KCl}]=0.1 \mathrm{M}$ for two membrane biases. The first case $(n-\mathrm{Si}$ biased at $V_{n}=-1 \mathrm{~V}, p-\mathrm{Si}$ at $V_{p}=0 \mathrm{~V}$ and electrolyte at $V=0 \mathrm{~V}$ ) is characterized by a single potential minimum in the pore, thus resulting in accumulation of cations in the nanopore center (Fig. 2A). The second case ( $n-\mathrm{Si}$ at $V_{n}=1 \mathrm{~V}, p-\mathrm{Si}$ at $V_{p}=0 \mathrm{~V}$ and electrolyte at $V=0 \mathrm{~V}$ ) gives rise to a potential profile with two extrema of opposite sign in the pore, which results in a dipolar ion charge in the pore: anions on the $n-\mathrm{Si}$ side, and cations on the $p-\mathrm{Si}$ side of the membrane (Fig. 2B).

For comparison with the $p-n$ membrane the potential variation in the nanopore of a single layered $n-$ Si membrane is present in Fig. 3. Depending on the applied potential bias dicated. $S_{1}$ is taken at the center of the nanopore, while $S_{2}$ is taken across the membrane, and parallel to the pore axis. The drawing is not to scale. (B) Biological channel in a polarized membrane
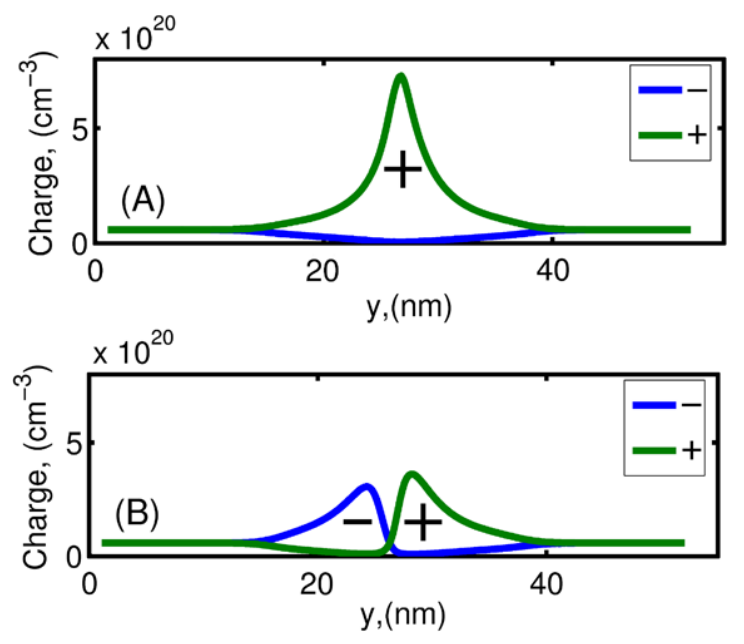

Fig. 2 Anion $\left(\mathrm{Cl}^{-}\right)$and cation $\left(\mathrm{K}^{+}\right)$concentrations in the electrolyte solution in the nanopore center at $S_{1}$ of a $p-n$ membrane for two membrane biases: (A) $V_{n}=-1 \mathrm{~V}$ and $V_{p}=0 \mathrm{~V}$; and (B) $V_{n}=1 \mathrm{~V}$ and $V_{p}=0 \mathrm{~V}$. Simulation parameters: $\sigma=-0.0256 \mathrm{C} / \mathrm{m}^{2}$, $N_{d}^{n}=2 \times 10^{20} \mathrm{~cm}^{-3}, N_{d}^{p}=2 \times 10^{20} \mathrm{~cm}^{-3},[\mathrm{KCl}]=0.1 \mathrm{M}$

to the membrane, a single potential maximum or potential minimum is present in the nanopore. In contrast to the $p-n$ membrane, single layered membrane potential results only in mono charge accumulation, either positive or negative, and never in bi-polar charge accumulation.

To calculate the current-voltage characteristics of the electrolyte we develop an electrodiffusion model for ionic transport similar to [11]. In short, we assume that the flux $J_{i}$ of species $i$ through the nanopore is described by the NernstPlanck equations.

$J_{i}=-D_{i} \frac{d c_{i}}{d x}-z_{i} D_{i} c_{i} \frac{F}{R T} \frac{d \phi}{d x}$,

where $D_{i}$ is the diffusion coefficient of species $i$ in the pore solution, and constants $F, R$ and $T$ have their usual meaning [12]. The total electrical current $I$ passing through the nanopore under the applied electrolyte bias $V=\phi_{L}-\phi_{R}$ (where $\phi_{L}$ and $\phi_{R}$ are potentials on the left and on the right 


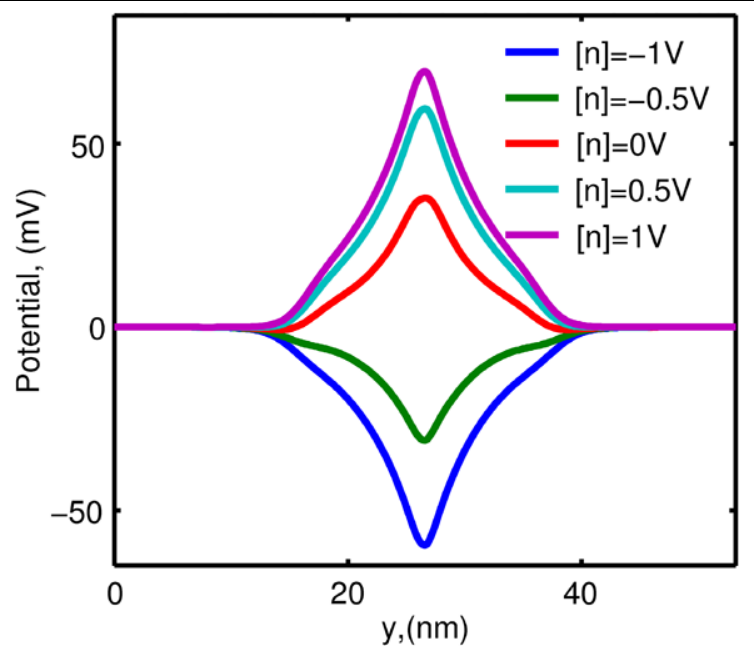

Fig. 3 Electrostatic potential in the nanopore for a $n-S i$ membrane, $N_{d}^{n}=2 \times 10^{20} \mathrm{~cm}^{-3}$, all other parameters are as in the $p-n$ membrane case, for various applied to the membrane potential biases

side of the membrane (they are equal when no driving bias is applied)) is given by

$I=F \pi r^{2} \sum_{i} z_{i} J_{i}$

and the bias potential difference applied to the system can be written

$V=-\left(\Delta \phi_{L}(V)+\Delta \phi_{D}(V)+\Delta \phi_{R}(V)\right)$.

$\Delta \phi_{L}$ and $\Delta \phi_{R}$ are the Donnan potential drops through the left and right interfaces, respectively, and $\Delta \phi_{D}=$ $\phi\left(d_{2}\right)-\phi\left(d_{1}\right)$ is the diffusion potential in the pore, between two points with coordinates $x=d_{1}$ and $x=d_{2}$. The potential differences $\Delta \phi_{L}, \Delta \phi_{D}$ and $\Delta \phi_{R}$ are determined directly from the calculated potential profiles in the nanopore.

Equations (1), (2), (3) with boundary conditions with appropriate boundary conditions allow us to obtain analytical expressions of ionic fluxes if we consider the constant field approximation between coordinates $x=d_{1}$ and $x=d_{2}$ which are chosen near the nanopore center:

$$
\begin{aligned}
J_{i}= & \frac{z_{i} F}{R T} \frac{D_{i} \Delta \phi_{D}}{\left(d_{2}-d_{1}\right)} \\
& \times\left[\frac{c_{i}\left(d_{1}\right) \exp \left(-z_{i} F \Delta \phi_{D} / R T\right)-c_{i}\left(d_{2}\right)}{1-\exp \left(-z_{i} F \Delta \phi_{D} / R T\right)}\right] .
\end{aligned}
$$

By assuming that the potential drops $\Delta \phi_{L}$ and $\Delta \phi_{R}$ at the left and right interface do not change with applied bias, as in biological channels [12], the $I-V$ characteristics can be obtained by substituting $\Delta \phi_{D}(V)=\Delta \phi_{D}(V=0)-V$ in the right hand side of (4) with $c_{S, L}=c_{S, R}=c_{0}=0.01 \mathrm{M}$ on each side of the membrane; we use $D_{\mathrm{K}^{+}}=1.95 \times$ $10^{-5} \mathrm{~cm}^{2} / \mathrm{s}, D_{\mathrm{Cl}^{-}}=2.03 \times 10^{-5} \mathrm{~cm}^{2} / \mathrm{s}$ for the diffusion
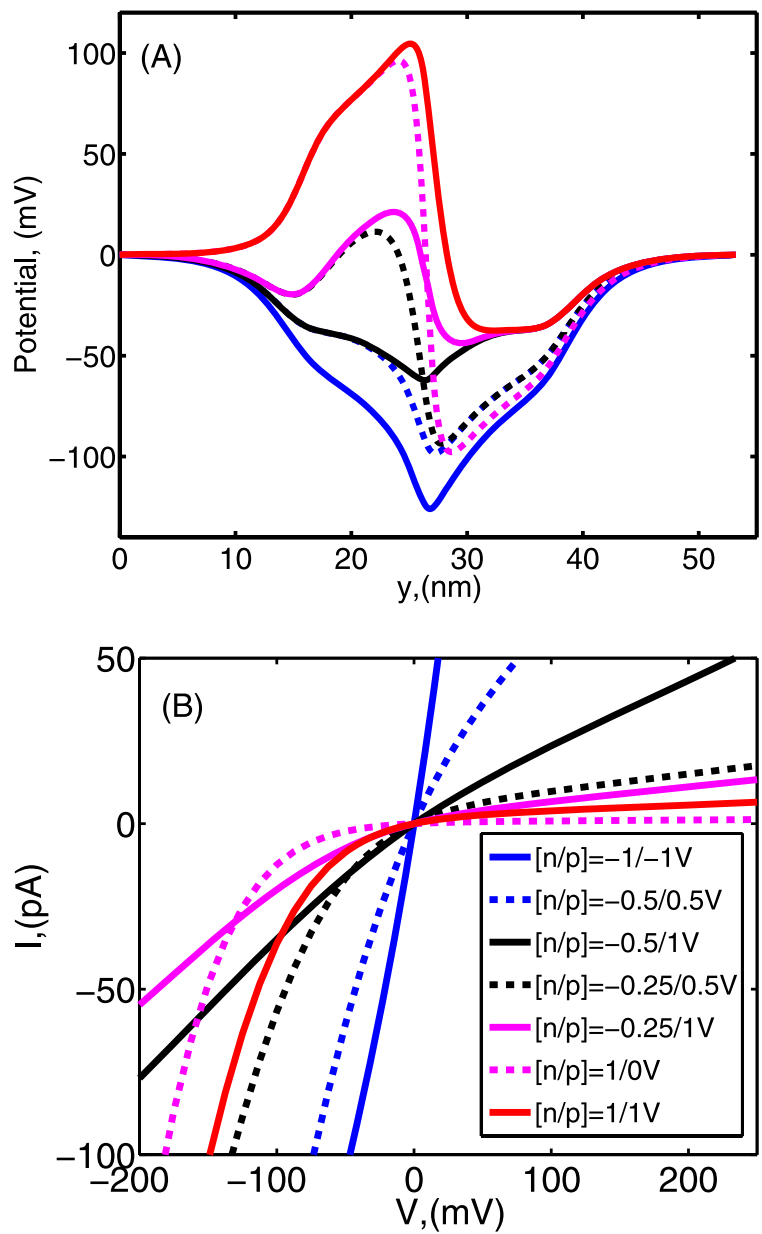

Fig. 4 (A) Electrostatic potential in the nanopore along cross-section $S_{1}$ for various membrane biases. $[\mathrm{KCl}]=0.01 \mathrm{M}$ and surface charge density $\sigma=-0.0256 \mathrm{C} / \mathrm{m}^{2}$ for $p-n$-membrane (doping densities are $N_{d}^{n}=2 \times 10^{20} \mathrm{~cm}^{-3}$ and $N_{d}^{p}=2 \times 10^{20} \mathrm{~cm}^{-3}$ ). Electrolyte bias $V=0$. Membrane $n$ - and $p$-layer bias potentials are indicated in legend in $(\mathbf{B})$ as $[n / p]$. (B) Current-voltage characteristics calculated for several membrane biases

coefficients of potassium and chlorine ions. Once the total electrical current is calculated, the nanopore conductance $G=d I / d V$ and the ion selectivity $S=\left|\frac{G_{\mathrm{Cl}}-G_{\mathrm{K}}}{G_{\mathrm{Cl}}+G_{\mathrm{K}}}\right|$ in the nanopore are readily obtained. More model details may be found in [10].

Figure 4A shows the electrostatic potential profiles in the nanopore for $[\mathrm{KCl}]=0.01 \mathrm{M}$ at different biases between the $n-\mathrm{Si}$ and $p-\mathrm{Si}$ sides of the semiconductor membrane at electrolyte bias $V=0$. The membrane potential biases $V_{n(p)}$ vary from $-1 \mathrm{~V}$ to $1 \mathrm{~V}$ with respect to the electrolyte. The potential variations in the nanopore are dramatic with up to $200 \mathrm{mV}$ magnitude. As a function of the voltage across the $p-n$ membrane, the $I-V$ characteristics behavior varies from quasi-ohmic (i.e. $V_{n}=-0.5 \mathrm{~V}, V_{p}=1 \mathrm{~V}$ ) to diodelike with vanishing leakage current at $V>0$ (i.e. $V_{n}=1 \mathrm{~V}$, $V_{p}=0$ V), Fig. 4B. 
The membranes under biases which result in nanopore potentials with double extrema of equal height lead to the low selectivity regime (i.e. $V_{n}=1 \mathrm{~V}, V_{p}=0 \mathrm{~V}$ ). The nanopore potentials with a dominant extrema result in the high selectivity regime. Thus, membranes with a single potential minimum is being selective towards positive $\left(\mathrm{K}^{+}\right)$ ions (i.e. $V_{n}=-1 \mathrm{~V}, V_{p}=-1 \mathrm{~V}$ ), and membranes with a large potential maximum is being selective towards negative $\left(\mathrm{Cl}^{-}\right)$ions (i.e. $V_{n}=1 \mathrm{~V}, V_{p}=1 \mathrm{~V}$ ).

\section{Conclusion}

In this work we showed that nanopore in a $p-n$ membrane can be tuned electrically from ohmic behavior to any desirable rectification, and to a complete blockade of the total ionic current. It can perform as ion filter with the possibility to filter ions of either sign. The $p-n$ membrane device mimics in a very basic way the operation of voltage gated biological channels in cells. The $p-n$ nanopore device also provides an opportunity to trap, stretch and effectively slow down DNA translocation in the pore, thus rising the resolution of the proposed nanopore sequencing device $[4,5]$. With semiconductor membranes it is also possible to add another $n$ or $p$ layer to create $n-p-n$ or $p-n-p$ structures that will allow control over the ionic flow in both directions, and this will be the topic of a forthcoming publication.
Acknowledgement This work was funded by NIH grant ROIHG003713-01. The authors gratefully acknowledge the use of the supercomputer time at the National Center for Supercomputer Applications.

\section{References}

1. Alberts, B., Bray, D., Johnson, A., Lewis, J., Raff, M., Roberts, K., Walter, P.: Essential Cell Biology. Garland (1998)

2. Beckstein, O., Sansom, M.: Phys. Biol. 1, 42-52 (2004)

3. Kasianowicz, J.J., Brandin, E., Branton, D., Deamer, D.W.: Proc. Natl. Acad. Sci. USA 93, 13770-13773 (1996)

4. Gracheva, M.E., Xiong, A., Aksimentiev, A., Schulten, K., Timp, G., Leburton, J.-P.: Nanotechnology 17, 622-633 (2006)

5. Heng, J.B., Ho, C., Kim, T., Timp, R., Aksimentiev, A., Grinkova, Y.V., Sligar, S., Schulten, K., Timp, G.: Biophys. J. 87, 2905-2911 (2004)

6. Karnik, R., Duan, C., Castelino, K., Daiguji, H., Majumdar, A.: Nano Lett. 7, 547-551 (2007)

7. Vlassiouk, I., Siwy, Z.S.: Nano Lett. 7, 552-556 (2007)

8. Press, W.H., Teukolsky, S.A., Vetterling, W.T., Flannery, B.P.: 2001. Numerical Recipes in Fortran 77. Cambridge University Press. On-line: http://www.nr.com

9. Gardner, C.L., Nonner, W., Eisenberg, R.S.: J. Comput. Electron. 3, 25-31 (2004)

10. Gracheva, M.E., Vidal, J., Leburton, J.-P.: Nanoletters 7(6), 17171722 (2007)

11. Ramírez, P., Mafé, S., Aguilella, V.M., Alcaraz, A.: Phys. Rev. E 68, 011910(1)-011910(8) (2003)

12. Lakshminarayanaiah, N.: Equations of Membrane Biophysics. Academic, New York (1984) 